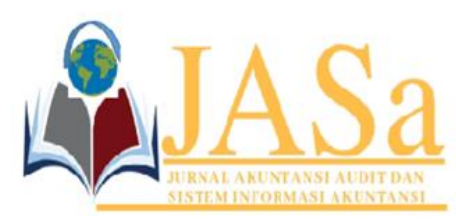

JASa (Jurnal Akuntansi, Audit dan Sistem Informasi Akuntansi)

Vol. 5 No.1/ April 2021

ISSN 2550-0732 print / ISSN 2655-8319 online

DOI; $10.36555 /$ jasa.v5i3.1624

\title{
ANALYSIS OF THE PROFITABILITY OF AGRICULTURAL SECTOR COMPANIES - SUB-SECTOR OF AGRICULTURE LISTED IN INDONESIA STOCK EXCHANGE
}

\author{
Tokit Masditok $^{*}$, Sriwardani ${ }^{2}$ \\ Universitas Langlangbuana, Indonesia \\ okitomas70@gmail.com ${ }^{* 1}$ fafasriwardani@gmail.com²
}

\begin{abstract}
This study aims to analyze the profitability of plantation sub-sector companies listed on the Indonesia Stock Exchange (BEI), based on the analysis of Gross Profit Margin (GPM), Nett Profit Margin (NPM), Return On Asset (ROA), and Return On Equity $(R O E)$. The data used in this research is secondary data in the form of financial statements of Plantation Sub-Sector Companies listed on the Indonesia Stock Exchange (IDX) for the period 2014-2018, which were obtained from www.idx.co.id. The method used in this research is the descriptive analysis based on quantitative/financial analysis. The results showed that companies with higher Total Assets and Total Equity have the potential to achieve better Total Revenue, thus supporting the achievement of better Earning After Tax (EAT). However, these conditions do not necessarily guarantee that the company will achieve the best Gross Profit Margin (GPM), Nett Profit Margin (NPM), Return On Asset (ROA), and Return On Equity (ROE) as well, given the best Gross Profit Margin (GPM), Nett Profit Margin (NPM), Return On Asset (ROA) and Return On Equity $(\mathrm{ROE})$ are financial ratios whose measurements are influenced by the width of the range or margin among other financial measures that underlie the calculation of these ratios.

Keyword: Gross Profit Margin (GPM), Nett Profit Margin (NPM), Return On Asset (ROA), Return On Equity (ROE).
\end{abstract}

\section{INTRODUCTION}

Indonesia as an agricultural country has positioned the agricultural sector as one of the sectors capable of providing an important role in economic activity. From the data collected since the preparation of this research proposal, data on the development of Indonesian plantation business, especially oil palm plantations concerning Indonesian Crude Palm Oil (CPO) Production, are obtained, including Agriculture Sector Contribution to Gross Domestic Product (GDP); as information obtained from www.bps.go.id (accessed February 6, 2019, 15.15 WIB), the Central Statistics Agency (BPS) informed that the contribution of the agricultural sector to GDP was quite large, namely around 13.14 percent in 2017 . One of the sub-sectors with considerable potential in the agricultural sector is the Plantation Subsector. The contribution of the Plantation Subsector to GDP is around 3.47 percent or first in the Agricultural Sector (BPS, Indonesian Palm Oil Statistics Publication 2017.3). Indonesia as the World's Largest Palm Oil Exporter; From the data presented by BPS, information can also be obtained that, in the Plantation Sub-Sector, oil palm is one of the plantation commodities that has made a positive contribution. Palm oil is one of Indonesia's export commodities as a foreign exchange earner, thus making Indonesia the world's largest producer and exporter of palm oil. In addition to the increasingly open export opportunities, the 


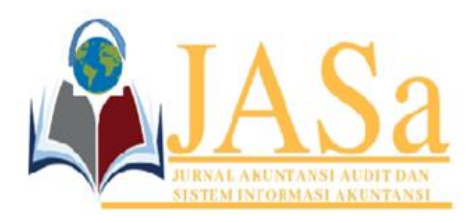

JASa (Jurnal Akuntansi, Audit dan Sistem Informasi Akuntansi)

Vol. 5 No.1/ April 2021

ISSN 2550-0732 print / ISSN 2655-8319 online

DOI; $10.36555 /$ jasa.v5i3.1624

domestic palm oil market is still quite large. Potential markets that will absorb the marketing of palm oil or CPO are the fractionation/manufacturing industry (especially the cooking oil industry), specialty fats (cocoa butter substitute), margarine/shortening, oleochemicals, and bath soap (BPS, Indonesian Palm Oil Statistics Publication 2017, 3).

Most of Indonesia's palm oil products are exported abroad and the rest is marketed domestically. Indonesia's palm oil exports reach five continents, namely Asia, Africa, Australia, America, and Europe with a major share in Asia. In 2017, the top five importing countries for Indonesian CPO were India, the Netherlands, Singapore, Italy and Spain. The volume of exports to India reached 4.63 million tons or 65.40 percent of the total export volume of Indonesian CPO with a value of US \$3,068 million. The second rank is the Netherlands, with an export volume of 0.62 million tonnes or 8.70 percent of Indonesia's total CPO volume with a value of US \$ 415.7 million. The third rank is Singapore, with an export volume of 0.60 million tonnes or 8.55 percent of Indonesia's total CPO export volume with a value of US \$ 398.6 million. The fourth rank is Italy with an export volume of 0.36 million tons or about 5.04 percent of the total export volume of Indonesian CPO with a value of US \$231.4 million. The fifth rank is Spain with an export volume of 0.22 million tonnes or 3.05 percent of the total export volume of CPO with a value of US \$138.6 million (BPS, Indonesian Palm Oil Statistics Publication 2017: 13). Development of Indonesian Palm Oil Area; BPS provided information that the area of oil palm plantations in Indonesia before 2017 during the last four years tended to show an increase, except in 2016 which experienced a decline. The increase ranged from 2.77 to 4.70 percent per year and decreased in 2016 by 0.52 percent. In 2014, Indonesia's oil palm plantation land was recorded at 10.47 million hectares, increasing to 11.26 million hectares in 2015 or an increase of 7.60 percent. In 2016, the total area of oil palm plantations decreased by 0.52 percent from 2015 to 11.20 million hectares. Furthermore, in 2017 the area of oil palm plantations is estimated to have increased by 9.80 percent from 2016 to 12.30 million hectares (BPS, Indonesian Palm Oil Statistics Publication 2017: 9). Status of Indonesian Oil Palm Plantation Entrepreneurs; According to the status of the concession, most of the oil palm plantations in 2016 were cultivated by Private Large Plantations (PBS), namely 5.75 million hectares (51.37\%), of which 4.74 million hectares $(42.31 \%)$ were cultivated by Smallholders (PR), and 0.71 million hectares (6.32\%) cultivated by State Large Plantations (PBN). In 2017, it is estimated that 6.05 million hectares $(49.17 \%)$ were cultivated by large private plantations (PBS), of which 5.61 million hectares (45.64\%) were cultivated by People's Plantation (PR), and 0.64 million hectares $(5.19 \%)$ cultivated by State Large Plantations (PBN) (BPS, Indonesian Palm Oil Statistics Publication 2017: 10). Indonesian CPO Oil Production; different from the area of oil palm, the development of Indonesian CPO production from 2013 to 2016 has always increased per year. From 2013 to 2015, palm oil production increased between 5.67 and 7.70 percent. Then in 2016, palm oil production experienced a sharp increase of 53.28 percent from 2015. In 2013 the production of CPO oil was 17.77 million tons, increasing to 31.49 million tons in 2016 , or an increase of 77.18 percent. While in 2017 it is estimated that CPO oil production will increase to 34.47 million tons or 9.46 percent (BPS, Indonesian Palm Oil Statistics Publication 2017: 10). Research objectives, namely the results of this study can prove that the company with a Value for Money; The highest Total Asset, Total Equity, Revenue, Cost of Revenue and Earning After Tax 


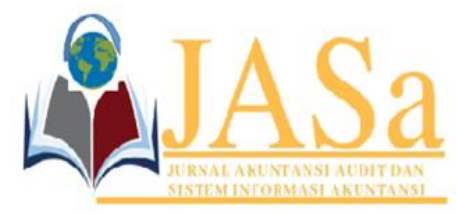

JASa (Jurnal Akuntansi, Audit dan Sistem Informasi Akuntansi)

Vol. 5 No.1/ April 2021

ISSN 2550-0732 print / ISSN 2655-8319 online

DOI; $10.36555 /$ jasa.v5i3.1624

(EAT) does not necessarily reach the percentage ratio; The highest Nett Profit margin (NPM), Gross Profit Margin (GPM), Return On Asset (ROA) and Return On Equity (ROE), considering the above ratios are determined more by how wide the company can maintain the range or margin between The elements in the financial statements which are used as elements in the formulation for calculating the ratios mentioned above.

A financial Statement (Financial Statement) is an overview that describes the financial performance of a company in a certain period. Where one of the financial analyzes that can be done to assess the company's financial performance is profitability analysis or formula to calculate the profitability ratio. Profitability ratios consist of two types of ratios, namely ratios that show a profit concerning sales, and ratios that show profits concerning investment (Harjitno 2012; 60). The Ratio that Shows Profit concerning Sales Gross Profit Margin (GPM); According to Harjitno (2012: 60) this ratio is the ratio of net sales minus cost of goods sold, to net sales, or the ratio between gross profit and net sales. Nett Profit Margin (NPM); According to Harjitno (2012: 60), this ratio shows the sales profit after calculating all expenses and income taxes. So this margin shows the ratio of net profit after tax to sales.

The ratio that shows the profit concerning investment

Return On Asset (RoA); According to Fahmi (2016: 82), this ratio looks at the extent to which the investment that has been invested can provide returns as expected. The formula used to calculate Return on Assets (RoA) is as follows: Return on Equity (RoE); According to Fahmi (2016: 82) this ratio shows the efficient use of own capital. The higher this ratio, the better. This ratio examines the extent to which a company uses its resources to be able to provide a return on equity.

\section{Data source}

\section{METHODS}

Where, the data used in this study were obtained from related official websites, as a secondary source. Data and Sources Data in this study consist of data and sources from the following: Data on Indonesian Palm Oil Statistics, obtained by accessing the official website of the Central Statistics Agency (BPS), namely www.bps.go.id. Data regarding the Agriculture Sector Companies in the Plantation Sector that were listed on the Indonesia Stock Exchange (IDX) for the period 2014-2018, was obtained by accessing the website www.sahamok.com. Financial Statements for Agriculture Sector Companies in the Plantation Sector that are listed on the Indonesia Stock Exchange (IDX) for the period 2014 - 2018, can be obtained by accessing the website www.idx.co.id. World CPO Price Movement, obtained by accessing the website www.palmoilanalytics.com.

Population

In this study, there is a population of 10 Plantation Sub-Sector Companies listed on the Indonesia Stock Exchange, as shown in the table as follows: 


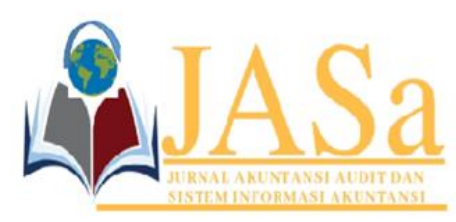

JASa (Jurnal Akuntansi, Audit dan Sistem Informasi Akuntansi)

Vol. 5 No.1/ April 2021

ISSN 2550-0732 print / ISSN 2655-8319 online

DOI;10.36555/jasa.v5i3.1624

Table 1. Population

\begin{tabular}{cclc}
\hline No. & $\begin{array}{c}\text { Share } \\
\text { Code }\end{array}$ & \multicolumn{1}{c}{ Name of Issuer } & Date of IPO \\
\hline 1 & AALI & Astra Agro Lestari Tbk & $09-12-1997$ \\
2 & ANJT & Austindo Nusantara Jaya Tbk & $10-05-2013$ \\
3 & BWPT & BW Plantation Tbk & $27-10-2009$ \\
4 & DSNG & Dharma Satya Nusantara Tbk & $14-06-2013$ \\
5 & GOLL & Golden Plantation Tbk & $23-12-2014$ \\
6 & GZCO & Gozco Plantation Tbk & $15-05-2008$ \\
7 & JAWA & Jaya Agra Wattie Tbk & $30-05-2011$ \\
8 & LSIP & PP London Sumatera Indonesia Tbk & $05-07-1996$ \\
9 & MAGP & Multi Agro Gemilang Plantation Tbk & $16-01-2013$ \\
10 & PALM & Provident Agro Tbk & $18-10-2012$ \\
\hline \multicolumn{4}{c}{ Source: www.sahamok.com (data processed) } \\
\hline
\end{tabular}

\section{Sample}

The sample selection using purposive sampling is because not all samples have the criteria following the authors specify, therefore the authors chose the purposive sampling technique. The sample criteria in this study are "Financial Statements Consisting of a Complete Balance Sheet and Income Statement During the 2014-108 Research Period". Where, of the total population of 10 (ten) companies in the Plantation Sub-Sector, there are only 8 (eight) companies that meet the criteria. So that MAGP and GOLL companies were excluded from the sample or not used as samples, because the financial statements of the two companies were not fully presented according to the sample criteria to meet the data analysis needs in this study.

\section{Descriptive Analysis}

Descriptive analysis in terms of evaluation of the independent variable and the dependent variable. Descriptive statistics are statistics used to analyze data by describing or describing the data that has been collected without references intended to be general or generalized (Sugiyono, 2017: 147). In this study, where there are only independent variables as the variables studied, a descriptive analysis will be carried out on the results of the financial analysis carried out on the balance sheet and income statement of the companies that are the research sample, namely the calculation results which include: Gross Profit Margin (GPM). )), Nett Profit Margin (NPM, Return on Asset (RoA), and Return on Equity (RoE), taking into account the movement of World Crude Palm Oil (CPO) Prices in 2014 - 2018.

\section{RESEARCH RESULTS AND DISCUSSION \\ Research result}

Development of Total Assets, Total Equity, Total Revenue, Cost of Revenue and Earning After Tax (EAT) of Plantation Sub-Sector Companies that have been listed on the Indonesia Stock Exchange (IDX), 2014 - 2018.

To answer the identification of the first problem in this research, namely "How the Development of Total Assets, Total Equity, Total Revenue, Cost of Revenue and Earning 


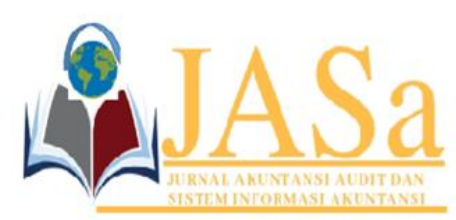

\section{JASa (Jurnal Akuntansi, Audit dan Sistem Informasi Akuntansi) \\ Vol. 5 No.1/ April 2021 \\ ISSN 2550-0732 print / ISSN 2655-8319 online \\ DOI; $10.36555 /$ jasa.v5i3.1624}

After Tax (EAT) of Plantation Sub-Sector Companies that have been listed on the Indonesia Stock Exchange (IDX), during the period 2014-2018 ", secondary data processing has been carried out, which is obtained through access on the website www.idx.co.id. The secondary data referred to is the Financial Statements of Plantation Sub-Sector Companies that have been listed on the Indonesia Stock Exchange (IDX), during the 2014-2018 period. Thus, from the results of secondary data processing mentioned above, research findings on Total Assets, Total Equity, Total Revenue, Cost of Revenue and Earning After Tax (EAT) can be obtained as follows:

\section{Total Assets of Companies in Plantation Sub-Sector 2014-2018}

The data is processed regarding the Total Assets of Companies in the Plantation Sub-Sector, during the 2014-2018 period or a total of 40 (forty) data, showing fluctuating figures, namely as follows:

Table 2. Total Assets of Companies in Plantation Sector 2014-2018

\begin{tabular}{|c|c|c|c|c|c|c|c|}
\hline \multirow{2}{*}{ No } & \multirow{2}{*}{ code } & \multicolumn{6}{|c|}{ Total Assets (Rp. Million) } \\
\hline & & 2014 & 2015 & 2016 & 2017 & 2018 & AVERAGE \\
\hline 1 & AALI & 18.558 .329 & 21.512 .371 & 24.226 .122 & 24.935 .426 & 26.835 .773 & 23.213.604 \\
\hline 2 & ANJT & 5.523 .726 & 6.895 .298 & 7.055 .347 & 7.675 .266 & 8.347 .759 & 7.099 .479 \\
\hline 3 & BWPT & 16.379 .840 & 17.658 .837 & 16.254 .353 & 15.991 .148 & 16.767.712 & 16.610 .378 \\
\hline 4 & DSNG & 5.921 .055 & 7.174 .488 & 7.853 .275 & 8.183 .318 & 8.336 .065 & 7.493 .640 \\
\hline 5 & GZCO & 3.232 .644 & 4.964 .076 & 3.547 .023 & 3.517 .586 & 3.277 .756 & 3.707 .817 \\
\hline 6 & JAWA & 3.062 .490 & 3.368 .152 & 3.291 .117 & 3.312 .482 & 3.489 .015 & 3.304 .651 \\
\hline 7 & LSIP & 8.655 .146 & 8.848 .792 & 9.459 .088 & 9.744 .381 & 10.427 .644 & 9.427 .010 \\
\hline 8 & PALM & 4.223 .636 & 4.696 .939 & 3.860 .776 & 2.849 .094 & 2.433 .865 & 3.612 .862 \\
\hline \multicolumn{2}{|c|}{ TOTAL } & 65.556 .866 & 75.118 .953 & 75.547.101 & 76.208 .701 & 79.915 .589 & 74.469 .442 \\
\hline
\end{tabular}

\section{Total Equities of Plantation Sub-Sector Companies 2014-2018}

The data is processed regarding the Total Equities of Companies in the Plantation Sub-Sector, during the 2014-2018 period or a total of 40 (forty) data, showing fluctuating figures, namely as follows: 


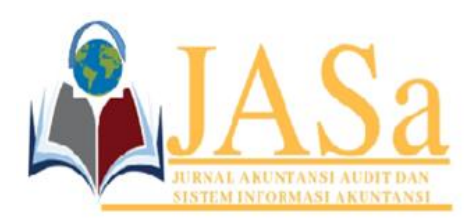

JASa (Jurnal Akuntansi, Audit dan Sistem Informasi Akuntansi)
Vol. 5 No.1/ April 2021

ISSN 2550-0732 print / ISSN 2655-8319 online

DOI;10.36555/jasa.v5i3.1624

Table 3. Total Equities of Companies in Plantation Sector 2014-2018

\begin{tabular}{|c|c|c|c|c|c|c|c|}
\hline \multirow{2}{*}{ No } & \multirow{2}{*}{ CODE } & \multicolumn{6}{|c|}{ Total Assets (Rp. Million) } \\
\hline & & 2014 & 2015 & 2016 & 2017 & 2018 & AVERAGE \\
\hline 1 & AALI & 11.837 .486 & 11.698 .787 & 17.593 .482 & 18.536 .438 & 18.920 .721 & 15.717 .383 \\
\hline 2 & ANJT & 4.670 .732 & 4.989 .681 & 4.765 .049 & 5.315 .994 & 5.635 .332 & 5.075 .358 \\
\hline 3 & BWPT & 6.946 .691 & 6.652 .915 & 6.259 .436 & 6.062 .693 & 6.260 .066 & 6.436 .360 \\
\hline 4 & DSNG & 1.678 .545 & 2.293 .331 & 2.507 .021 & 2.704 .341 & 3.249 .739 & 2.486 .595 \\
\hline 5 & GZCO & 1.554 .303 & 2.670 .016 & 1.144 .507 & 1.537 .980 & 1.369 .013 & 1.655 .164 \\
\hline 6 & JAWA & 1.315 .658 & 1.289 .941 & 1.050 .319 & 839.979 & 736.747 & 1.046 .529 \\
\hline 7 & LSIP & 7.218 .834 & 7.337 .978 & 7.645 .984 & 8.122 .165 & 8.167 .479 & 7.698 .488 \\
\hline 8 & PALM & 1.680 .456 & 1.687 .264 & 2.326 .314 & 1.540 .310 & 1.666 .978 & 1.780 .264 \\
\hline \multicolumn{2}{|c|}{ TOTAL } & 36.902 .705 & 38.619 .913 & 43.292 .112 & 44.659 .900 & 46.006 .075 & 41.896 .141 \\
\hline
\end{tabular}

\section{Total Company Revenues in Plantation Sector 2014-2018}

The data is processed regarding the Total Revenues of Companies in the Plantation Sub-Sector, during the 2014-2018 period or a total of 40 (forty) data, showing a fluctuating number, namely as follows:

Table 4. Total Company Revenues in Plantation Sector 2014-2018

\begin{tabular}{|c|c|c|c|c|c|c|c|}
\hline \multirow{2}{*}{ No } & \multirow{2}{*}{ CODE } & \multicolumn{6}{|c|}{ Total Revenues (Rp. Million) } \\
\hline & & 2014 & 2015 & 2016 & 2017 & 2018 & AVERAGE \\
\hline 1 & AALI & 16.305 .831 & 13.059 .216 & 14.121 .374 & 17.305 .688 & 9.021 .481 & 13.962 .718 \\
\hline 2 & ANJT & 2.083 .238 & 1.954 .333 & 1.806 .380 & 2.192 .030 & 1.005 .042 & 1.808 .205 \\
\hline 3 & BWPT & 2.264 .396 & 2.674 .271 & 2.541 .763 & 3.045 .954 & 1.404 .372 & 2.386 .151 \\
\hline 4 & DSNG & 3.842 .182 & 4.898 .479 & 4.425 .060 & 3.942 .024 & 5.159 .911 & 4.453.531 \\
\hline 5 & GZCO & 462.840 & 491.605 & 544.884 & 646.945 & 283.246 & 485.904 \\
\hline 6 & JAWA & 760.611 & 658.309 & 590.138 & 555.140 & 354.113 & 583.662 \\
\hline 7 & LSIP & 4.726 .539 & 4.189 .615 & 3.847 .869 & 4.738 .022 & 1.763 .272 & 3.853 .063 \\
\hline 8 & PALM & 1.057 .576 & 1.046 .536 & 1.169 .778 & 759.995 & 306.697 & 868.116 \\
\hline \multicolumn{2}{|c|}{ TOTAL } & 31.503 .213 & 28.972.364 & 29.047 .246 & 33.185 .798 & 19.298.134 & 28.401 .351 \\
\hline
\end{tabular}




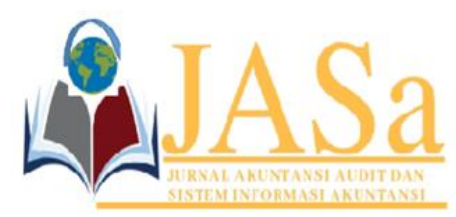

JASa (Jurnal Akuntansi, Audit dan Sistem Informasi Akuntansi)

Vol. 5 No.1/ April 2021

ISSN 2550-0732 print / ISSN 2655-8319 online

DOI;10.36555/jasa.v5i3.1624

\section{Cost of Revenues of Companies in Plantation Sector, 2014-2018}

The data is processed regarding the Cost of Revenues of Companies in the Plantation Sub-Sector, during the 2014-2018 period or a total of 40 (forty) data, showing a fluctuating number, as follows:

Table 5. Cost of Revenues Companies in Plantation Sector, 2014-2018

\begin{tabular}{|c|c|c|c|c|c|c|c|}
\hline \multirow{2}{*}{ No } & \multirow{2}{*}{ CODE } & \multicolumn{6}{|c|}{ Cost of Revenues (Rp. Million) } \\
\hline & & 2014 & 2015 & 2016 & 2017 & 2018 & AVERAGE \\
\hline 1 & AALI & 11.354 .037 & 9.977 .118 & 10.445 .360 & 13.160 .438 & 7.370 .143 & 10.461 .419 \\
\hline 2 & ANJT & 1.701 .382 & 1.946 .161 & 1.197 .915 & 1.512 .640 & 694.927 & 1.410 .605 \\
\hline 3 & BWPT & 1.635 .264 & 2.100 .500 & 1.948 .273 & 2.276 .709 & 1.000 .481 & 1.792 .245 \\
\hline 4 & DSNG & 2.651 .763 & 3.189 .046 & 3.250 .076 & 2.993 .149 & 3.436 .965 & 3.104 .200 \\
\hline 5 & GZCO & 324.962 & 381.189 & 687.781 & 603.345 & 341.218 & 467.699 \\
\hline 6 & JAWA & 544.718 & 511.343 & 520.568 & 493.697 & 335.047 & 481.075 \\
\hline 7 & LSIP & 3.090 .100 & 3.073 .774 & 2.737 .084 & 3.395 .184 & 1.367 .404 & 2.732.709 \\
\hline 8 & PALM & 703.261 & 719.071 & 835.338 & 507.181 & 227.290 & 598.428 \\
\hline \multicolumn{2}{|c|}{ TOTAL } & 22.005 .487 & 21.898.202 & 21.622.395 & 24.942.343 & 14.773.475 & 21.048 .380 \\
\hline
\end{tabular}

\section{Earning After Tax of Companies in Plantation Sector, 2014-2018}

The data is processed regarding Earning After Tax of Companies in the Plantation Sub-Sector, during the 2014-2018 period or a total of 40 (forty) data, showing fluctuating figures, namely as follows:

Table 6. Earning After Tax Perusahaan Sub Sektor Perkebunan Tahun 2014-2018

\begin{tabular}{|c|c|c|c|c|c|c|c|}
\hline \multirow{2}{*}{ No } & \multirow{2}{*}{ CODE } & \multicolumn{6}{|c|}{ EAT (Rp. Million) } \\
\hline & & 2014 & 2015 & 2016 & 2017 & 2018 & AVERAGE \\
\hline 1 & AALI & 2.621 .275 & 695.684 & 2.114.299 & 2.113 .629 & 815.241 & 1.672 .026 \\
\hline 2 & ANJT & 188.362 & (122.920) & 123.601 & 642.169 & 2.058 & 166.654 \\
\hline 3 & BWPT & 194.638 & $(181.400)$ & (391.367) & (187.791) & 2.015 & (112.781) \\
\hline 4 & DSNG & 215.696 & 649.794 & 302.519 & 252.040 & 671.046 & 418.219 \\
\hline 5 & GZCO & 51.007 & 31.816) & $(1.547 .604)$ & (168.518) & $(126.741)$ & (364.734) \\
\hline 6 & JAWA & 51.686 & (11.716) & (225.133) & (199.929) & (89.043) & $(94.827)$ \\
\hline 7 & LSIP & 916.695 & 623.309 & 592.769 & 763.423 & 224.036 & 624.046 \\
\hline 8 & PALM & 168.152 & $(55.242)$ & 219.100 & 68.216 & 851 & 80.215 \\
\hline \multicolumn{2}{|c|}{ TOTAL } & 4.407 .511 & 1.565 .693 & 1.188.184 & 3.283 .239 & 1.499 .463 & 2.388 .818 \\
\hline
\end{tabular}

Submitted: April 19, 2021; Accepted: April 19, 2021;

Published: April 24, 2021; Website: http://journalfeb.unla.ac.id/index.php/jasa 


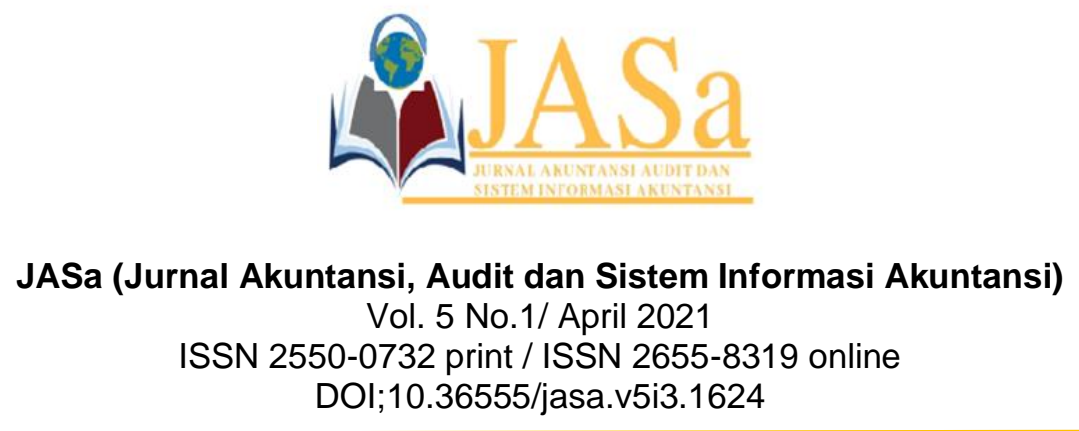

The development of Gross Profit Margin (GPM), Nett Profit Margin (NPM), Return on Asset (RoA), and Return on Equity (RoE), which were achieved by plantation sub-sector companies that have been listed on the Indonesia Stock Exchange (IDX), 2014 - 2018.

From the data that has been obtained above, the next calculation is carried out, to answer the identification of the second problem of this study, namely "How many GPM, NPM, ROA, and RoE, have been achieved by the companies in the Plantation SubSector that have been listed in Indonesia Stock Exchange (IDX), 2014 - 2018 ". So that the results/research findings regarding the calculation/percentage of GPM, NPM, ROA, and RoE are obtained, as follows:

\section{Gross Profit Margin (GPM) of Plantation Sub-Sector Companies listed on the IDX, 2014-2018.}

The data is processed regarding the analysis of financial performance regarding the GPM of Companies in the Plantation Sub-Sector, during the 2014-2018 period or a total of 40 (forty) data, showing fluctuating values, namely as follows:

Table 7. Gross Profit Margin (GPM) of Plantation Sub-Sector Companies listed on the IDX

\begin{tabular}{cccccccc}
\hline \multirow{2}{*}{ No } & \multirow{2}{*}{ CODE } & $\mathbf{2 0 1 4}$ & $\mathbf{2 0 1 5}$ & $\mathbf{2 0 1 6}$ & $\mathbf{2 0 1 7}$ & $\mathbf{2 0 1 8}$ & AVERAGE \\
\hline \hline 1 & AALI & $30,37 \%$ & $23,60 \%$ & $26,03 \%$ & $23,95 \%$ & $18,30 \%$ & $\mathbf{7 4 , 9 2 \%}$ \\
2 & ANJT & $18,33 \%$ & $0,42 \%$ & $33,68 \%$ & $30,99 \%$ & $30,86 \%$ & $\mathbf{7 8 , 0 1 \%}$ \\
3 & BWPT & $27,78 \%$ & $21,46 \%$ & $23,35 \%$ & $25,25 \%$ & $28,76 \%$ & $\mathbf{7 5 , 1 1 \%}$ \\
4 & DSNG & $30,98 \%$ & $34,90 \%$ & $26,55 \%$ & $24,07 \%$ & $33,39 \%$ & $\mathbf{6 9 , 7 0 \%}$ \\
5 & GZCO & $29,79 \%$ & $22,46 \%$ & $-26,23 \%$ & $6,74 \%$ & $-20,47 \%$ & $\mathbf{9 6 , 2 5 \%}$ \\
6 & JAWA & $28,38 \%$ & $22,32 \%$ & $11,79 \%$ & $11,07 \%$ & $5,38 \%$ & $\mathbf{8 2 , 4 2 \%}$ \\
7 & LSIP & $34,62 \%$ & $26,63 \%$ & $28,87 \%$ & $28,34 \%$ & $22,45 \%$ & $\mathbf{7 0 , 9 2 \%}$ \\
8 & PALM & $33,50 \%$ & $31,29 \%$ & $28,59 \%$ & $33,27 \%$ & $25,89 \%$ & $\mathbf{6 8 , 9 3 \%}$ \\
& TOTAL & $\mathbf{6 9 , 8 5 \%}$ & $\mathbf{7 5 , 5 8 \%}$ & $\mathbf{7 4 , 4 4 \%}$ & $\mathbf{7 5 , 1 6 \%}$ & $\mathbf{7 6 , 5 5 \%}$ & $\mathbf{7 4 , 3 2 \%}$ \\
\hline \multicolumn{7}{c}{ source: www.idx.co.id (data processed) } \\
\hline
\end{tabular}

Nett Profit Margin (NPM) of Plantation Sub-Sector Companies listed on the IDX, 2014-2018.

The data is processed regarding the analysis of financial performance regarding the NPM of Plantation Sub Sector Companies, during the 2014-2018 period or a total of 40 (forty) data, showing a fluctuating value, namely as follows: 


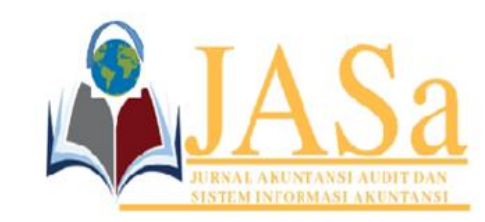

JASa (Jurnal Akuntansi, Audit dan Sistem Informasi Akuntansi)

Vol. 5 No.1/ April 2021

ISSN 2550-0732 print / ISSN 2655-8319 online

DOI;10.36555/jasa.v5i3.1624

Table 8. Nett Profit Margin (NPM) of Plantation Sub-Sector Companies listed on the IDX

\begin{tabular}{|c|c|c|c|c|c|c|c|}
\hline \multirow{2}{*}{ No } & \multirow{2}{*}{ CODE } & \multicolumn{6}{|c|}{ NPM (\%) } \\
\hline & & 2014 & 2015 & 2016 & 2017 & 2018 & AVERAGE \\
\hline 1 & AALI & $16,08 \%$ & $5,33 \%$ & $14,97 \%$ & $12,21 \%$ & $9,04 \%$ & $11,97 \%$ \\
\hline 2 & ANJT & $9,04 \%$ & $-6,29 \%$ & $6,84 \%$ & $29,30 \%$ & $0,20 \%$ & $9,22 \%$ \\
\hline 3 & BWPT & $8,60 \%$ & $-6,78 \%$ & $-15,40 \%$ & $-6,17 \%$ & $0,14 \%$ & $-4,73 \%$ \\
\hline 4 & DSNG & $5,61 \%$ & $13,27 \%$ & $6,84 \%$ & $6,39 \%$ & $13,00 \%$ & $9,39 \%$ \\
\hline 5 & GZCO & $11,02 \%$ & $-6,47 \%$ & $-284,02 \%$ & $-26,05 \%$ & $-44,75 \%$ & $-75,06 \%$ \\
\hline 6 & JAWA & $6,80 \%$ & $-1,78 \%$ & $-38,15 \%$ & $-36,01 \%$ & $-25,15 \%$ & $-16,25 \%$ \\
\hline 7 & LSIP & $19,39 \%$ & $14,88 \%$ & $15,41 \%$ & $16,11 \%$ & $12,71 \%$ & $16,20 \%$ \\
\hline 8 & PALM & $15,90 \%$ & $-5,28 \%$ & $18,73 \%$ & $8,98 \%$ & $0,28 \%$ & $9,24 \%$ \\
\hline \multicolumn{2}{|c|}{ TOTAL } & $13,99 \%$ & $5,40 \%$ & $4,09 \%$ & $9,89 \%$ & $7,77 \%$ & $8,23 \%$ \\
\hline
\end{tabular}

Return on Asset (RoA) of Plantation Sub-Sector Companies listed on the IDX, 2014-2018.

The data is processed regarding the analysis of financial performance regarding RoA of companies in the Plantation Sub-Sector, during the 2014-2018 period or a total of 40 (forty) data, showing fluctuating values, as follows:

Table 9. Return on Asset (RoA) of Plantation Sub-Sector Companies listed on the IDX

\begin{tabular}{cccccccc}
\hline \multirow{2}{*}{ No } & \multirow{2}{*}{ CODE } & $\mathbf{6}$ & \multicolumn{5}{c}{ ROA (\%) } \\
& & $\mathbf{2 0 1 4}$ & $\mathbf{2 0 1 5}$ & $\mathbf{2 0 1 6}$ & $\mathbf{2 0 1 7}$ & $\mathbf{2 0 1 8}$ & AVERAGE \\
\hline \hline 1 & AALI & $14,12 \%$ & $3,23 \%$ & $8,73 \%$ & $8,48 \%$ & $3,04 \%$ & $\mathbf{7 , 2 0 \%}$ \\
2 & ANJT & $3,41 \%$ & $-1,78 \%$ & $1,75 \%$ & $8,37 \%$ & $0,02 \%$ & $\mathbf{2 , 3 5 \%}$ \\
3 & BWPT & $1,19 \%$ & $-1,03 \%$ & $-2,41 \%$ & $-1,17 \%$ & $0,01 \%$ & $-0,68 \%$ \\
4 & DSNG & $3,64 \%$ & $9,06 \%$ & $3,85 \%$ & $3,08 \%$ & $8,05 \%$ & $\mathbf{5 , 5 8 \%}$ \\
5 & GZCO & $1,58 \%$ & $-0,64 \%$ & $-43,63 \%$ & $-4,79 \%$ & $-3,87 \%$ & $-9,84 \%$ \\
6 & JAWA & $1,69 \%$ & $-0,35 \%$ & $-6,84 \%$ & $-6,04 \%$ & $-2,55 \%$ & $-\mathbf{2 , 8 7 \%}$ \\
7 & LSIP & $10,59 \%$ & $7,04 \%$ & $6,27 \%$ & $7,83 \%$ & $2,15 \%$ & $\mathbf{6 , 6 2 \%}$ \\
8 & PALM & $3,98 \%$ & $-1,18 \%$ & $5,68 \%$ & $2,39 \%$ & $0,03 \%$ & $\mathbf{2 , 2 2 \%}$ \\
& TOTAL & $\mathbf{6 , 7 2 \%}$ & $\mathbf{2 , 0 8 \%}$ & $\mathbf{1 , 5 7 \%}$ & $\mathbf{4 , 3 1 \%}$ & $\mathbf{1 , 8 8 \%}$ & $\mathbf{3 , 3 1 \%}$ \\
\hline \multicolumn{7}{c}{ source: www.idx.co.id (data processed) } \\
\end{tabular}

Submitted: April 19, 2021; Accepted: April 19, 2021;

Published: April 24, 2021; Website: http://journalfeb.unla.ac.id/index.php/jasa 


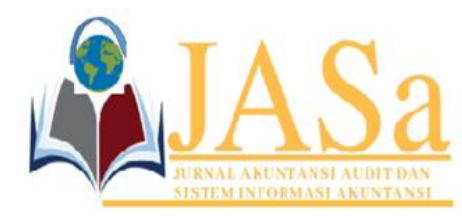

JASa (Jurnal Akuntansi, Audit dan Sistem Informasi Akuntansi)

Vol. 5 No.1/ April 2021

ISSN 2550-0732 print / ISSN 2655-8319 online

DOI;10.36555/jasa.v5i3.1624

Return on Equity (RoE) of Plantation Sub-Sector Companies listed on the IDX, 2014-2018.

The data is processed regarding the analysis of financial performance regarding RoE of companies in the Plantation Sub-Sector, during the 2014-2018 period or a total of 40 (forty) data, showing a fluctuating value, namely as follows:

Table 10. Return on Equity (RoE) of Plantation Sub-Sector Companies listed on the IDX

\begin{tabular}{cccccccc}
\hline \multirow{2}{*}{ No } & CODE & $\mathbf{5}$ & $\mathbf{5}$ & \multicolumn{3}{c}{ ROE (\%) } \\
& & $\mathbf{2 0 1 4}$ & $\mathbf{2 0 1 5}$ & $\mathbf{2 0 1 6}$ & $\mathbf{2 0 1 7}$ & $\mathbf{2 0 1 8}$ & AVERAGE \\
\hline \hline 1 & AALI & $22,14 \%$ & $5,95 \%$ & $12,02 \%$ & $11,40 \%$ & $4,31 \%$ & $\mathbf{1 0 , 6 4 \%}$ \\
2 & ANJT & $4,03 \%$ & $-2,46 \%$ & $2,59 \%$ & $12,08 \%$ & $0,04 \%$ & $\mathbf{3 , 2 8 \%}$ \\
3 & BWPT & $2,80 \%$ & $-2,73 \%$ & $-6,25 \%$ & $-3,10 \%$ & $0,03 \%$ & $-\mathbf{1 , 7 5 \%}$ \\
4 & DSNG & $12,85 \%$ & $28,33 \%$ & $12,07 \%$ & $9,32 \%$ & $20,65 \%$ & $\mathbf{1 6 , 8 2 \%}$ \\
5 & GZCO & $3,28 \%$ & $-1,19 \%$ & $-135,22 \%$ & $-10,96 \%$ & $-9,26 \%$ & $-\mathbf{2 2 , 0 4 \%}$ \\
6 & JAWA & $3,93 \%$ & $-0,91 \%$ & $-21,43 \%$ & $-23,80 \%$ & $-12,09 \%$ & $-\mathbf{9 , 0 6 \%}$ \\
7 & LSIP & $12,70 \%$ & $8,49 \%$ & $7,75 \%$ & $9,40 \%$ & $2,74 \%$ & $\mathbf{8 , 1 1 \%}$ \\
8 & PALM & $10,01 \%$ & $-3,27 \%$ & $9,42 \%$ & $4,43 \%$ & $0,05 \%$ & $\mathbf{4 , 5 1 \%}$ \\
& TOTAL & $\mathbf{1 1 , 9 4 \%}$ & $\mathbf{4 , 0 5 \%}$ & $\mathbf{2 , 7 4 \%}$ & $\mathbf{7 , 3 5 \%}$ & $\mathbf{3 , 2 6 \%}$ & $\mathbf{5 , 8 7 \%}$ \\
\hline \multicolumn{7}{c}{ source: www.idx.co.id (data processed) } \\
\end{tabular}

\section{Discussion}

Development of Total Assets, Total Equity, Total Revenue, Cost of Revenue and Earning After Tax (EAT) of Plantation Sub-Sector Companies that have been listed on the Indonesia Stock Exchange (IDX), 2014 - 2018.

Total Assets

Total assets managed by the eight companies in the period 2014-2018, an average of Rp. 74,469,442,000,000, -. The largest average total assets owned by AALI company, which is an average of Rp. $23,213,604,000,000$, - or $31.17 \%$ of the average total assets of the company. While the lowest is owned by JAWA companies, with an average of Rp. 3,304,651,000,000, - or $4.44 \%$ of the average total assets of the entire company. A graphic illustration to illustrate the Average Total Assets managed by each company in 2014-2018, can be presented in a bar graph as follows: 


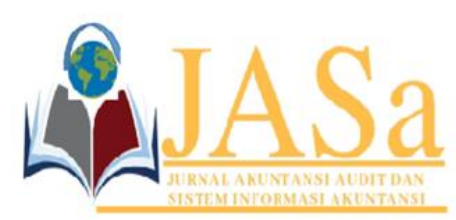

JASa (Jurnal Akuntansi, Audit dan Sistem Informasi Akuntansi)

Vol. 5 No.1/ April 2021

ISSN 2550-0732 print / ISSN 2655-8319 online

DOI;10.36555/jasa.v5i3.1624

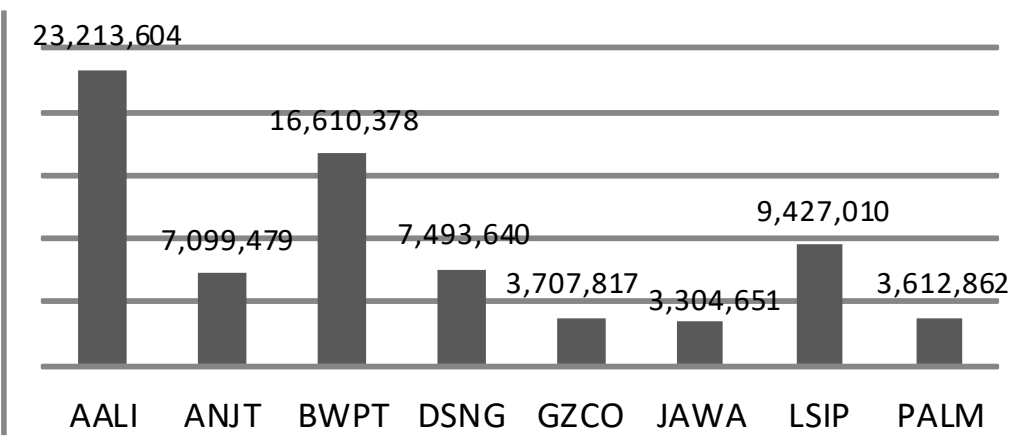

Figure 1. Average Total Assets (IDR Million) source: www.idx.co.id (data processed)

\section{Total Equity}

Total equity managed by the eight companies in the 2014-2018 period, averaged Rp. $41,896,141,000,000$, -. As with the average Total Assets, the largest average Total Equity is also owned by AALI company, which is an average of Rp. 15,717,383,000,000, - or $37.52 \%$ of the average Total Equity of the company. Meanwhile, the lowest average Total Equity is owned by JAVA companies, which is an average of Rp. $1,046,529,000,000$, - or $2.50 \%$ of the average Total Equity of the whole company. A graphic illustration to illustrate the average Total Equity managed by each company, for the period 2014-2018, can be presented in a bar graph as follows:

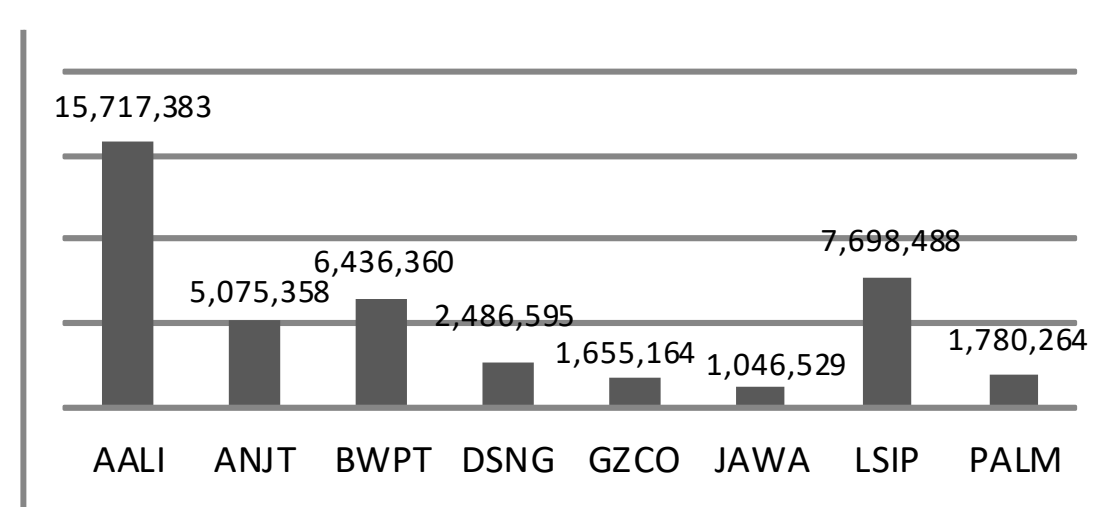

Figure 2. Average total equity source: www.idx.co.id (data processed)

\section{Total Revenue}

The total Revenues achieved by the eight companies in the period 2014-2018 reached an average of Rp. 28,401,351,000, -. The largest total revenue achieved by AALI company, which is an average of Rp. $13,962,718,000,000$, - every year, so this also shows that in the period 2014-2018, AALI company controlled $49.16 \%$ of the average Total Revenues achieved by the eight companies. Meanwhile, GZCO company experienced the lowest Total Revenues, with an average of Rp. 485,904,000,000, - every year or only $1.71 \%$ of the average Total Revenues achieved by the eight companies. A 


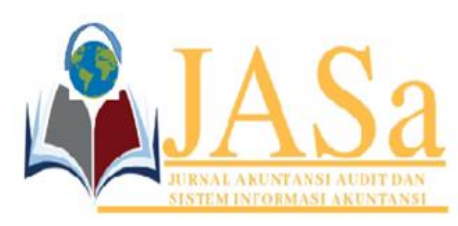

\section{JASa (Jurnal Akuntansi, Audit dan Sistem Informasi Akuntansi) \\ Vol. 5 No.1/ April 2021 \\ ISSN 2550-0732 print / ISSN 2655-8319 online \\ DOI;10.36555/jasa.v5i3.1624}

graphic illustration to illustrate the Average Total Revenues achieved by each company, for the period 2014-2018, can be presented in a bar graph as follows:

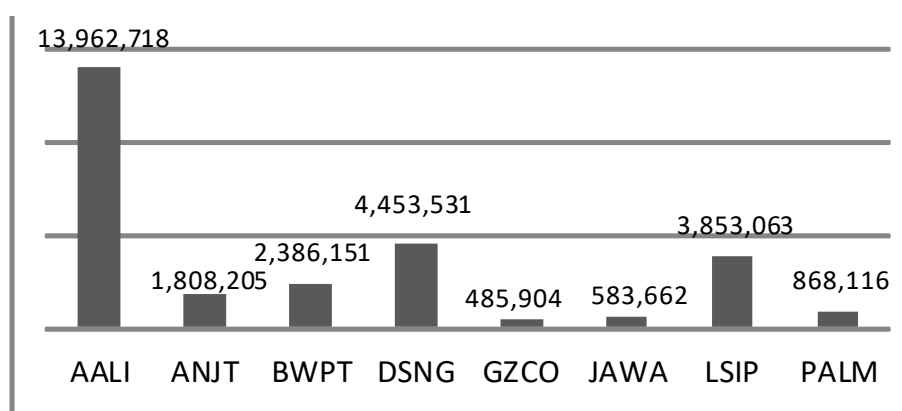

Figure 3. Average total revenue in 2014-2015 source: www.idx.co.id (data processed)

\section{Cost of Revenue}

Cost of Revenues experienced by the eight companies in the period 2014-2018, the largest experienced by AALI companies, namely an average of Rp. $10,461,419,000,000$, - annually. Meanwhile, GZCO company achieved the lowest, with an average of Rp. 467,699,000,000, - annually. A graphic illustration to illustrate the Average Cost of Revenues achieved by each company, for the period 2014-2018, can be presented in a bar graph as follows:

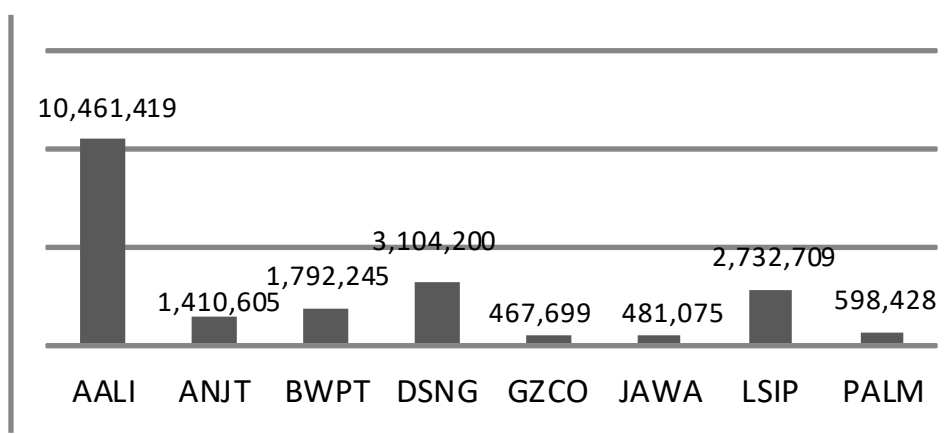

Figure 4. The average cost of revenues 2014-2018 source: www.idx.co.id (data processed)

\section{Earning After Tax (EAT)}

EAT was achieved by the eight companies in the period 2014-2018, the largest was achieved by AALI company, with an average EAT of Rp. 1,672,026,000,000, - every year where this is proportional to the average Total Revenue achieved by AALI which is also the highest as described in the previous sub-chapter. Meanwhile, the GZCO company experienced the lowest EAT, with an average loss (minus) of -Rp. $364,734,000,000$, - annually. A graphic illustration to illustrate the Average Cost of Revenues achieved by each company, for the period 2014-2018, can be presented in a bar graph as follows: 


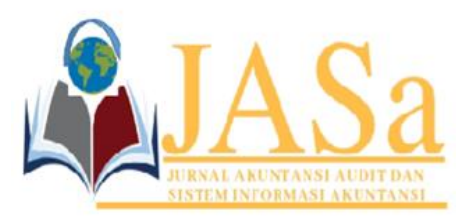

JASa (Jurnal Akuntansi, Audit dan Sistem Informasi Akuntansi)

Vol. 5 No.1/ April 2021

ISSN 2550-0732 print / ISSN 2655-8319 online

DOI;10.36555/jasa.v5i3.1624

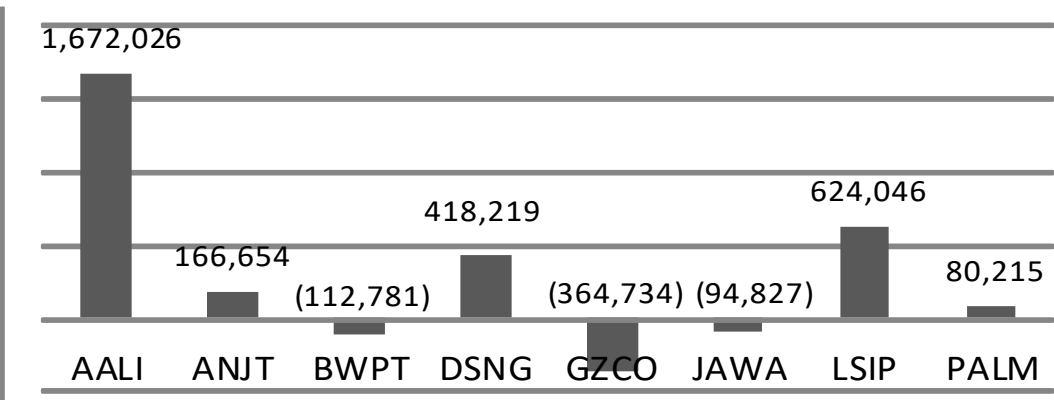

Figure 5. Average 2014-2018 earning after tax source: www.idx.co.id (data processed)

The development of Gross Profit Margin (GPM), Nett Profit Margin (NPM), Return on Asset (RoA), and Return on Equity (RoE), which were achieved by plantation subsector companies that have been listed on the Indonesia Stock Exchange (IDX), 2014 2018.

\section{Gross Profit Margin (GPM)}

The average GPM percentage of the eight companies during the 2014-2018 period reached an average total of $74.32 \%$. The company that achieved the highest average GPM was GZCO, which was an average of $96.25 \%$ or $129.52 \%$ of the total average GPM of all companies. Meanwhile, the companies that achieved the lowest average GPM were experienced by PALM companies, namely an average of $68.93 \%$ or $92.76 \%$ of the total average GPM of all companies. A graphic illustration to illustrate the average GPM achieved by the eight companies compared to the total average GPM of the eight companies during the period 2014-2018, can be presented in the bar graph as follows:

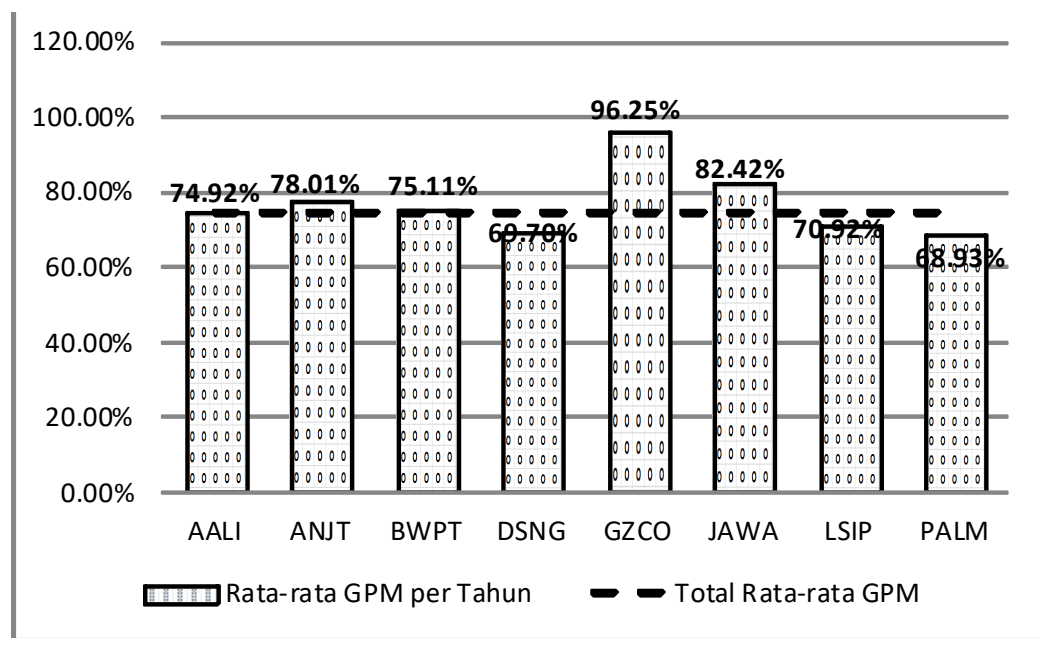

Figure 6. Average GMP Company / the Year 2014-2018 source: www.idx.co.id (data processed) 


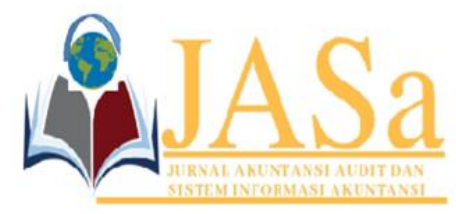

JASa (Jurnal Akuntansi, Audit dan Sistem Informasi Akuntansi)

Vol. 5 No.1/ April 2021

ISSN 2550-0732 print / ISSN 2655-8319 online

DOI; $10.36555 /$ jasa.v5i3.1624

The achievement of the average GPM figure is formed by the average Total Revenue and the Average Cost of Revenue achieved by each of the above companies. GZCO as a company that achieves the highest GPM, has an average Total Revenue of Rp. $485,904,000,000$, - with an average Cost of Revenue of Rp. 467,699,000,000, -. Meanwhile, the PALM company, as the company with the lowest average GPM, only had an average Total Revenue of Rp. 868,116,000,000, - with an average Cost of Revenue of Rp. 598,428,000,000, However, the highest and lowest attainments regarding the average Total Revenue and Cost of Revenue as the elements that make up the average GPM, have a composition of companies that achieve it differently. Where, companies that achieve the highest or lowest average GPM, do not necessarily have the highest or the lowest average Total Revenue and average Cost of Revenue. Where for the highest average Total Revenue achieved by AALI company, which reached an average of Rp. $13,962,718,000,000$, -. Meanwhile, the lowest average total revenue was experienced by the company GZCO with an average figure of Rp. 485,904,000,000, -. For the highest average Cost of Revenue experienced by AALI company, reaching an average of Rp. $10,461,419,000,000$, -. Meanwhile, the lowest average Cost of Revenue is achieved by the company GZCO, with an average figure of Rp. 467,699,000,000, - A graphic illustration to illustrate the average Total Revenue and the Average Cost of Revenue achieved by all companies as a forming factor for the average GPM of each company during the 2014-2018 period, can be presented in the bar graph as follows:

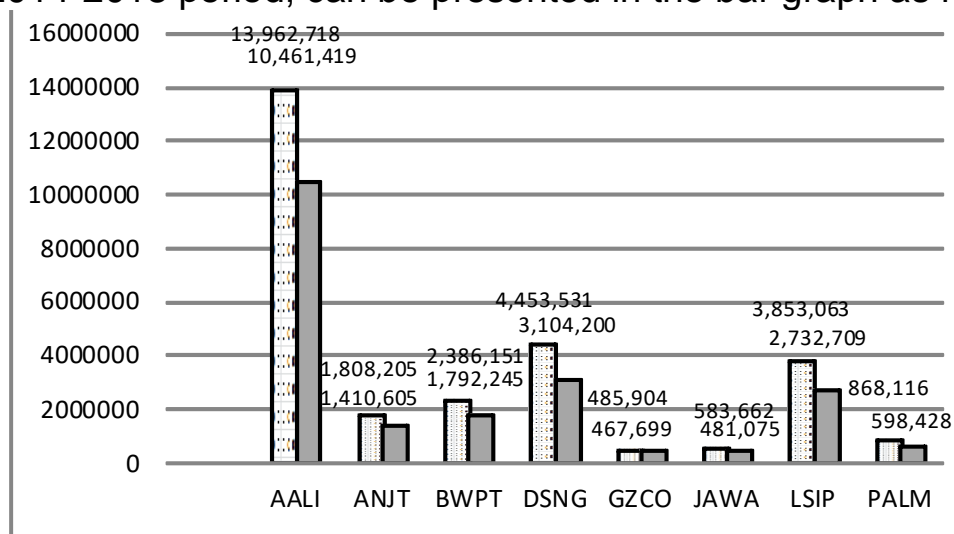

Figure 7. Average Total Revenue and Cost Revenue 2014-2018 source: www.idx.co.id (data processed)

So from the descriptive descriptions above, conclusions can also be drawn regarding the achievement of the average percentage of GPM in the 2014-2018 period, as follows: Although AALI company achieved the highest average Total Revenue of Rp. 13,962,718,000,000, - with an average Cost of Revenue of Rp. 10,461,419,000,000, -, where the average GPM was achieved at $74.92 \%$. However, the highest average GPM percentage during the 2014-2018 period was achieved by the GZCO company, which was an average of $96.25 \%$ or $129.52 \%$ of the total average GPM of all companies of $74.32 \%$. . This shows that, although the company GZCO only achieved an average revenue of Rp. $485,904,000,000$, - or only controlling $1.71 \%$ of the average Total 


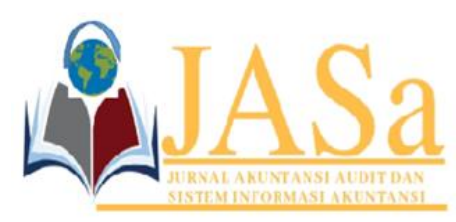

JASa (Jurnal Akuntansi, Audit dan Sistem Informasi Akuntansi)

Vol. 5 No.1/ April 2021

ISSN 2550-0732 print / ISSN 2655-8319 online

DOI;10.36555/jasa.v5i3.1624

Revenue achieved by the eight companies of Rp. $28,401,351,000,000$, -, but the company GZCO was able to create a wide enough range or margin between the average revenue of Rp. 485,904,000,000, - with an average Cost of Revenue of Rp. 467,699, -, to achieve an average GPM of $96.25 \%$ mentioned above. AALI company which in terms of rupiah value can generate the largest average revenue of Rp. 13,962,718,000,000, or controlling $49.16 \%$ or controlling almost half of the average Total Revenue achieved by the eight companies of Rp. $28,401,351,000,000$, -, was only able to achieve an average GPM of $74.92 \%$. This happens because the range or margin between average Revenue and the Average Cost of Revenue achieved by AALI is not wider than that GZCO company. The achievement of the average GPM percentage is strongly influenced by how much the company's ability to create a range or margin between the average revenue and the Average Cost of Revenue. The wider the range or margin between the average Revenue and the Average Cost of Revenue can be maintained, the greater the average percentage of GPM that can be achieved.

\section{Nett Profit Margin (NPM)}

The average NPM percentage of the eight companies during the 2014-2018 period reached an average total of $8.23 \%$. The company that achieved the highest NPM rate was LSIP, which reached an average figure of $16.20 \%$ or $196.80 \%$ of the total average NPM of the eight companies. Meanwhile, companies that achieved the lowest NPM and even minus were experienced by the GZCO company, which reached an average minus figure of $-75.06 \%$ or $-912.09 \%$ of the total average NPM of all companies. The minus NPM experienced by the GZCO company is inversely proportional to the GZCO achievement, as described in the previous sub-chapter, where in terms of GPM achievement, the GZCO company is the company that achieves the highest GPM percentage. A graphic illustration to illustrate the average NPM achieved by the eight companies compared to the Total Average NPM of the eight companies for the period 2014-2018, can be presented in the bar graph as follows:

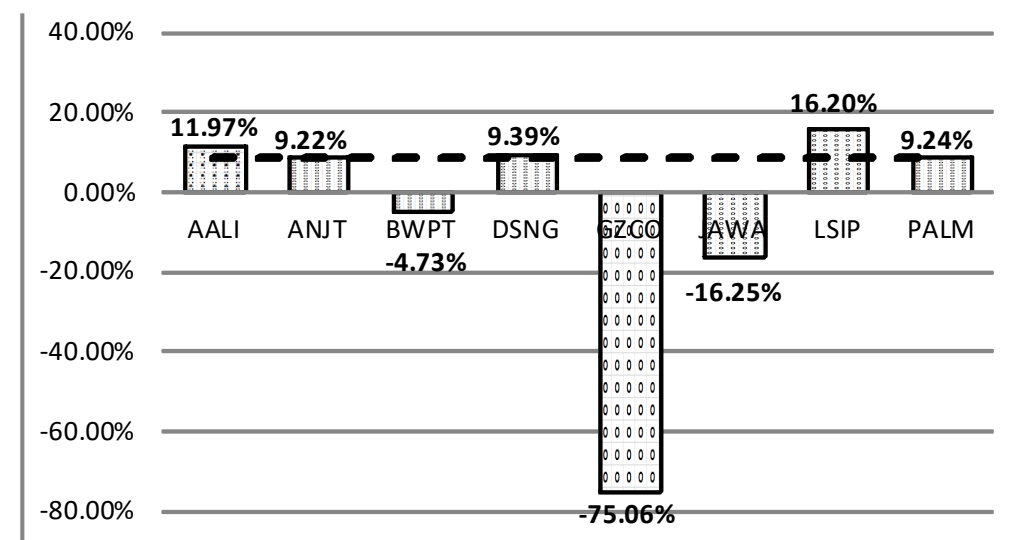

Figure 8. Average NPM of companies in 2014-2018 source:www.idx.co.id (data processed) 


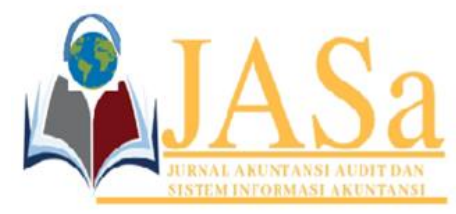

\author{
JASa (Jurnal Akuntansi, Audit dan Sistem Informasi Akuntansi) \\ Vol. 5 No.1/ April 2021 \\ ISSN 2550-0732 print / ISSN 2655-8319 online \\ DOI;10.36555/jasa.v5i3.1624
}

The achievement of the average NPM figure is formed by the average Total Revenue and the aforementioned average EAT achieved by each company. LSIP as a company that achieves the highest NPM, has an average Total Revenue of Rp. $3,853,063,000,000$, - with an average EAT of Rp. 624,046,000,000, -. Meanwhile, GZCO, as the company with the lowest NPM, only has an average Total Revenue of Rp. $485,904,000,000$, - with an average EAT, which is a minus average of -Rp. $364,734,000,000$, -. However, the highest and lowest achievements regarding the average Total Revenue and EAT as the elements that make up the average NPM, have a composition of companies that achieve it differently. Where, companies that achieve the highest or lowest NPM, do not necessarily have the highest or lowest Total Revenue and EAT as well. Where for the highest average Total Revenue, achieved by the company AALI, which reached an average figure of $\mathrm{Rp} .13,962,718,000,000$, - . Meanwhile, the lowest average total revenue was experienced by the company GZCO with an average figure of Rp. 485,904,000,000, -. For the highest average EAT achieved by AALI company, namely reaching an average of Rp. 1,672,026..000,000, -. Meanwhile, the lowest EAT was experienced by GZCO (Gozco Plantation Tbk) with an average EAT minus an average of Rp. 364,734,000,000, - A graphic illustration to illustrate the average Total Revenue and the average EAT achieved by all companies as a forming factor for the average NPM of each company for the 2014-2018 period, can be presented in the bar graph as follows:

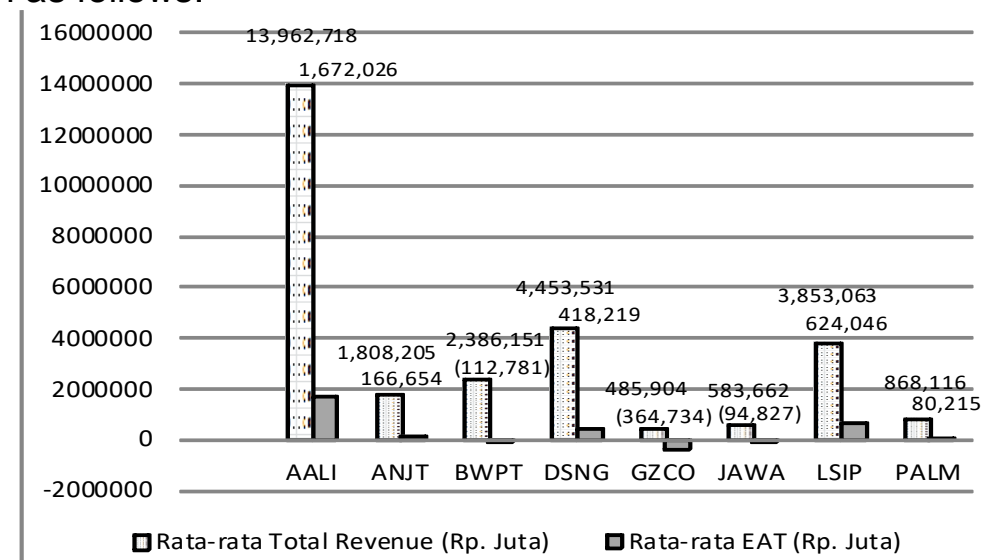

Figure 9. Average total revenue and average EAT source: www.idx.co.id (data processed)

So from the descriptive descriptions above, conclusions can also be drawn regarding the achievement of the average percentage of NPM in the 2014-2018 period, as follows: Although AALI company achieved the highest average Total Revenue of Rp. $13,962,718,000,000$, - with an average EAT of Rp. 1,672,026,000,000, - where the average NPM was achieved at $11.97 \%$. However, the highest average percentage of NPM in the eight companies during the 2014-2018 period was achieved by LSIP companies, namely an average of $16.20 \%$ or $196.80 \%$ above the total average NPM of all companies ". The foregoing shows that, although LSIP companies only reach an average Total Revenue of Rp. $3,853,063,000,000$, - or only controlling $13.57 \%$ of the average Total Revenue achieved by the eight companies of Rp. $28,401,351,000,000$, -, but the LSIP company was able to create 


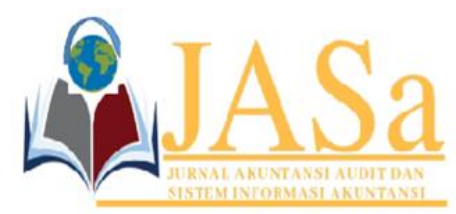

JASa (Jurnal Akuntansi, Audit dan Sistem Informasi Akuntansi)

Vol. 5 No.1/ April 2021

ISSN 2550-0732 print / ISSN 2655-8319 online

DOI; $10.36555 /$ jasa.v5i3.1624

a wide enough range or margin between the average Total Revenue of Rp. $3,853,063,000,000$, - with an average EAT of Rp. 624,046,000,000, -, thus achieving an average NPM figure of $16.20 \%$ mentioned above. This also means that $83.80 \%$ of the average Total Revenue achieved by LSIP is cost as a deduction factor for revenue or company revenue. AALI company which in terms of rupiah value can generate the highest average Total Revenue, namely Rp. $13,962,718,000,000$, - or controlling $49.16 \%$ or controlling almost half of the average Total Revenue achieved by the eight companies of Rp. $28,401,351,000,000$, -, was only able to achieve an average NPM of $11.97 \%$. This happens because the range or margin between the average Total Revenue and the average EAT achieved by AALI is not wider than that of LSIP companies. Wherewith an average Total Revenue of Rp. 13,962,718,000,000, - LSIP companies only achieve an average NPM of $11.97 \%$ or an average EAT of Rp. $1,672,026,000,000$, - The average percentage of NPM achievement is greatly influenced by how much the company's ability to creates the range or margin between the average Total Revenue and the average EAT. The wider the range or margin between the average Total Revenue and the average EAT can be maintained, the greater the average percentage of NPM that can be achieved.

\section{Return on Asset (ROA)}

The average percentage of ROA of the eight companies during the 2014-2018 period reached a total average of $3.31 \%$. The company that achieved the highest average ROA was AALI, which was an average of $7.20 \%$ or $217.41 \%$ of the total average ROA of all companies. Meanwhile, companies with the lowest average ROA and even minus were experienced by GZCO companies with an average of $-9.84 \%$ or $-296.92 \%$ of the total average ROA of all companies. A graphic illustration to illustrate the average ROA achieved by the eight companies compared to the total average ROA of the eight companies for the period 2014-2018, can be presented in a bar graph as follows:

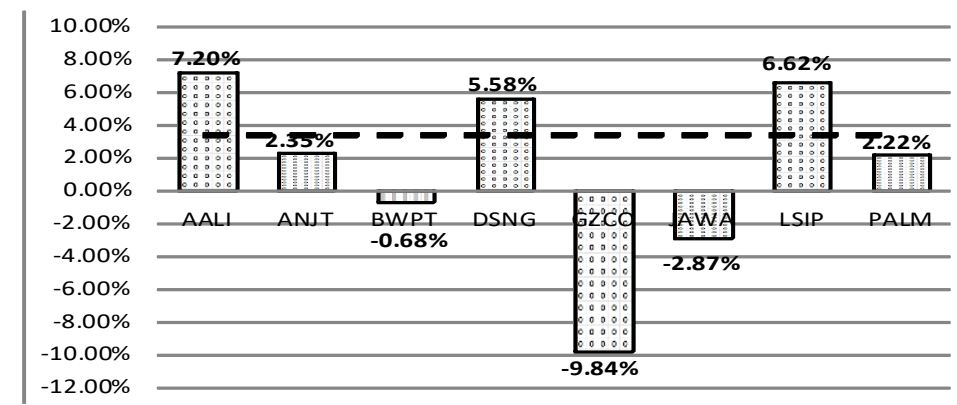

Figure 10. Average ROA of the Company in 2014-2018 source: www.idx.co.id (data processed)

The achievement of the average ROA is formed by the average total assets and the average EAT achieved by each of the above companies. AALI as a company that achieves the highest average ROA, has an average Total Asset of Rp. 23,213,604,000,000, - with an EAT of Rp. 1,672,026,000,000, -. Meanwhile, GZCO companies have average total assets of Rp. 3,707,817,000,000, - with an average EAT of Rp. 364,734,000,000, -. However, the 


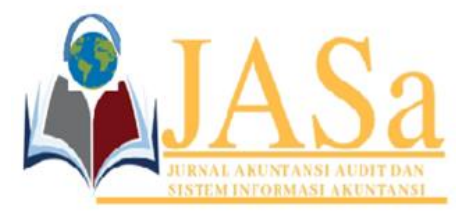

\author{
JASa (Jurnal Akuntansi, Audit dan Sistem Informasi Akuntansi) \\ Vol. 5 No.1/ April 2021 \\ ISSN 2550-0732 print / ISSN 2655-8319 online \\ DOI; $10.36555 /$ jasa.v5i3.1624
}

highest and lowest attainments regarding the average Total Asset and the average EAT as elements forming the average ROA, have a composition of companies that achieve them differently. Where, companies that achieve the highest or lowest average ROA, do not necessarily have an average Total Asset and reach the highest or lowest average EAT as well. Where the highest average total asset is still owned by AALI company which reaches an average of Rp. $23,213,604,000,000$, - . While the lowest average total assets owned by JAWA companies with an average figure of Rp. 3,304,651,000,000, -. For the highest average EAT was also achieved by AALI company, namely reaching an average of Rp. $1,672,026 . .000,000$, - . Meanwhile, the lowest EAT was experienced by GZCO with an average EAT minus an average of -Rp. 364,734,000,000, - A graphic illustration to illustrate the average total assets and average EAT achieved by all companies as a forming factor for the average ROA of each company for the period 2014-2018, can be presented in the bar graph as follows:

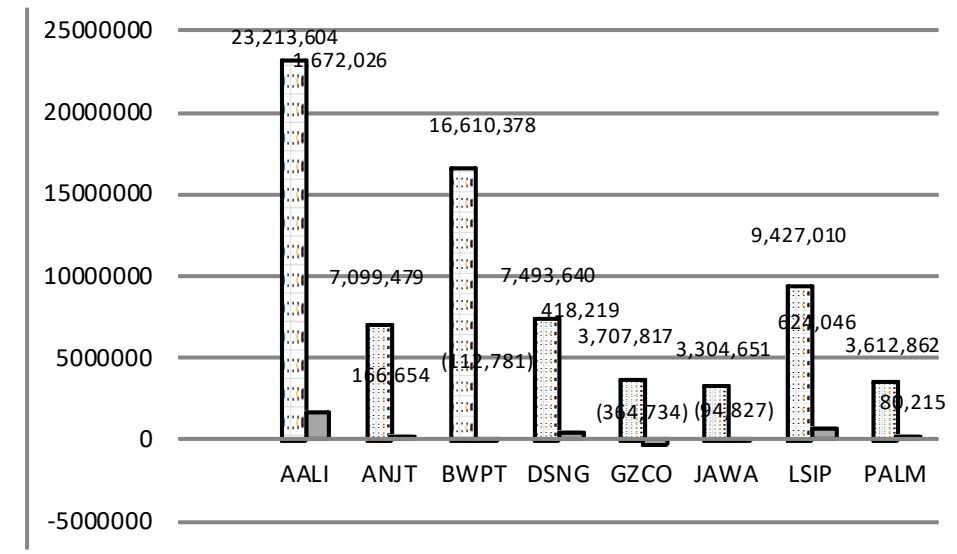

Figure 11. Average total assets and average EAT 2014-2018 source: www.idx.co.id (data processed)

So from the descriptive descriptions above, conclusions can also be drawn regarding the achievement of the average percentage of Return on Assets (ROA) in the 2014-2018 period, as follows: AALI company has average total assets, on average EAT, and the highest average ROA among the eight companies. The average total assets owned by AALI is Rp. 23,213,604,000,000, -, with an average EAT of Rp. $1,672,026 . .000,000$, -, and an average ROA of $7.20 \%$. With the largest average total assets, it is only natural that AALI company is also able to achieve the largest average EAT, so it is also fitting that the company is finally able to achieve the largest average ROA as well. This also shows that AALI company has been quite effective in using the average total assets of its companies to be used to produce an average EAT. So that the achievement of the average percentage of ROA is greatly influenced by how much the company's ability to produce a comparison of the average EAT to the average total assets owned. In the context of its comparison to the average total assets, the greater the average EAT obtained, the greater the average percentage of ROA that can be achieved by the company.

\title{
Return on Equity (ROE)
}




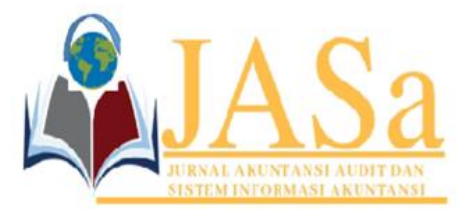

JASa (Jurnal Akuntansi, Audit dan Sistem Informasi Akuntansi)

Vol. 5 No.1/ April 2021

ISSN 2550-0732 print / ISSN 2655-8319 online

DOI;10.36555/jasa.v5i3.1624

The average percentage of ROE of the eight companies during the 2014-2018 period reached an average total of $8.23 \%$. The company that achieved the highest average ROE was DSNG company, which achieved an average ROE of $16.82 \%$ or $286.49 \%$ of the total average ROE of all companies. Meanwhile, companies with the lowest average ROE and even minus were experienced by GZCO companies with an average of $-22.04 \%$ or $-375.36 \%$ of the total average ROE of all companies. A graphic illustration to illustrate the average ROE achieved by the eight companies compared to the total average of the eight companies for the period 2014-2018, can be presented in the bar graph as follows:

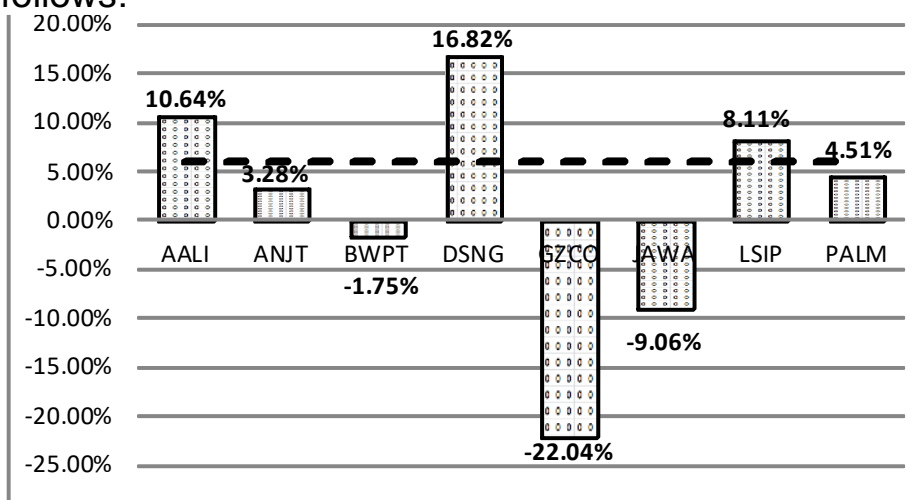

Figure 12. Average ROE

source: www.idx.co.id (data processed)

The achievement of the average ROE is formed by the average EAT and the average Total Equity achieved by each company mentioned above. DSNG as a company that achieves the highest average ROE, has an average EAT of Rp. 418,219,000,000, - with an average Total Equity of Rp. 2,486,595,000,000, -. Meanwhile, GZCO companies have an average EAT minus Rp. 364,734,000,000, - with an average Total Equity of Rp. $1,655,164,000,000$, However, the highest and lowest attainments regarding the average EAT and the average Total Equity as elements forming the average ROE, have a composition of companies achieving them differently. Where, companies that achieve the highest or lowest average ROE, do not necessarily reach the EAT average and reach the highest or lowest average Total Asset as well. Where the highest average EAT was achieved by AALI company, namely reaching an average of Rp. 1,672,026..000,000, -. Meanwhile, the lowest EAT was experienced by GZCO with an average EAT minus an average of -Rp. $364,734,000,000$, - For the average Total Equity, the highest is owned by AALI, which is an average of Rp. 15,717,383,000,000, -. While the lowest average Total Equity is owned by JAWA with an average Total Equity of Rp. 1,046,529,000,000, - A graphic illustration to illustrate the average Total Equity and average EAT owned by all companies as a forming factor for the average ROE of each company for the period 2014-2018, can be presented in the bar graph as follows: 


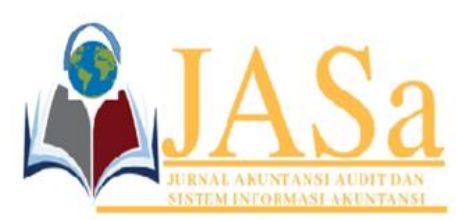

JASa (Jurnal Akuntansi, Audit dan Sistem Informasi Akuntansi)

Vol. 5 No.1/ April 2021

ISSN 2550-0732 print / ISSN 2655-8319 online

DOI; $10.36555 /$ jasa.v5i3.1624

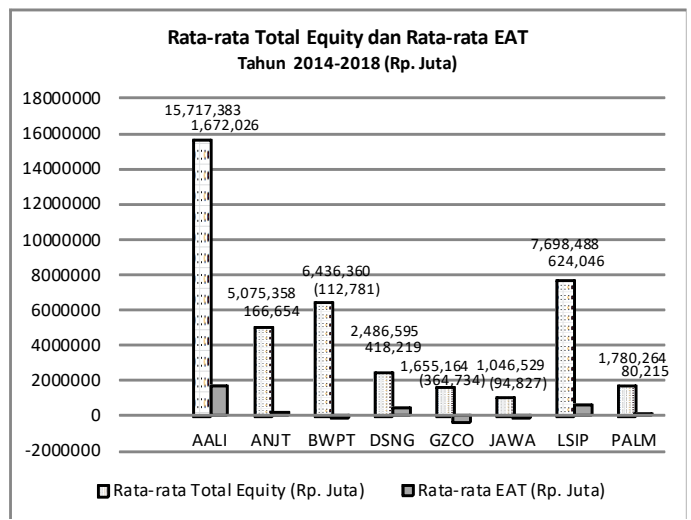

Figure 13. Average of Total Equity and EAT

source: www.idx.co.id (data processed)

So from the descriptive descriptions mentioned above, it can be concluded that the average ROE percentage achievement in the 2014-2018 period is that; Although AALI company has the highest average Total Equity of Rp. 15,717,383,000,000, - with an average EAT of Rp. 1,672,026,000,000, - where the average ROE was achieved at $10.64 \%$. However, the highest average percentage of ROE during the 2014-2018 period was achieved by the DSNG company, which was an average of $16.82 \%$ or $286.49 \%$ above the total average ROE of all companies ". The above shows that with an average Total Equity owned is Rp. 2,486,595,000,000, -, the DSNG company was able to achieve an average EAT of Rp. 418,219,000,000, -, to be able to achieve an average ROE of $16.82 \%$, which is the highest average ROE among the eight companies. This also shows that the DSNG company has been quite effective in using the average Total Equity provided by shareholders, to be used in generating the average EAT. So that the achievement of the average percentage of ROE is greatly influenced by how much the company's ability to produce a comparison of the average EAT to the average Total Equity owned. In the context of its comparison to the average Total Equity, the greater the average EAT obtained, the greater the percentage of average ROE that can be achieved by the company.

\section{CONCLUSION}

The development of Total Assets, Total Equity, Total Revenue, and Cost of Revenue owned by Plantation Sub-Sector Companies that have been listed on the Indonesia Stock Exchange (IDX), during the period 2014 - 2018, has fluctuated. Where Total Assets, Total Equity, Total Revenue, Cost of Revenue have contributed to the fluctuations in EAT formation that can be achieved by each company. Of the eight companies, where AALI company is the company that has the largest average Total Asset and Average Total Equity, it is easier for AALI company (Astra Agro Lestari Tbk) to achieve the largest average Total Revenue, so AALI company during 2014-2018 it reached the highest average EAT as well. The development of GPM, NPM, ROA, and ROE achieved by Plantation Sub-Sector Companies that have been listed on the Indonesia Stock Exchange (IDX), during the period 2014 - 2018, also fluctuated. This occurs as a result of the fluctuations in the development of Total Assets, Total Equity, 


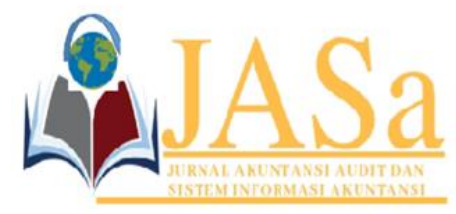

JASa (Jurnal Akuntansi, Audit dan Sistem Informasi Akuntansi)

Vol. 5 No.1/ April 2021

ISSN 2550-0732 print / ISSN 2655-8319 online

DOI; $10.36555 /$ jasa.v5i3.1624

Total Revenue, Cost of Revenue, and EAT mentioned above. Although the AALI company is a company with the largest average Total Asset, Total Equity, Total Revenue, and EAT during the 2014-2018 period, this does not mean AALI company or a company experiencing financial conditions similar to AALI (Astra Agro Lestari Tbk) will achieve the highest percentage of GPM and NPM as well. This happens because the percentage of GPM and the percentage of NPM are not only formed by the amount of Rupiah Total Revenue, Cost of Revenue, and EAT, but rather emphasize the extent to which the company can reach the range or margin between the three. The wider the range or margin between Total Revenue and Cost of Revenue, the higher the GPM percentage will be. Meanwhile, the wider the range or margin between Total Revenue and EAT, the higher the percentage of NPM will be. Where, In this study, companies that achieve the highest average percentage of GPM are companies (Gozco Plantation Tbk). Meanwhile, companies that achieve the highest average percentage of NPM are LSIP companies (PP London Sumatra Indonesia Tbk). As a company that had the largest average total assets during the 2014-2018 period, AALI was the company that achieved the largest average Total Revenue and average EAT as well. This also makes AALI a company that achieves the highest average percentage of ROA as well. can be achieved by AALI companies as well. However, this did not occur with the average ROE percentage, where the highest average percentage of ROE was achieved by DSNG companies. Where, this happens because in the formation of ROA and ROE percentages, companies are required to be able to create a large ratio of EAT to Total Asset in achieving ROA, as well as a large comparison of EAT to Total Equity in achieving ROE. The greater the EAT achieved, the better the ratio level of EAT to Total Assets and to Total Equity.

\section{REFERENCES}

Harjito, Agus, (2012), Manajemen Keuangan, Edisi Kedua, Cetakan Kedua, Jakarta : Ekonisia.

Sartono, Agus, (2008), Manajemen Keuangan Teori dan Aplikasi, $\quad$ Edisi Keempat, Yogyakarta : BPFE.

Gede Erni Sulindawati, Ni Luh, (2016), Manajemen Depok : Rajawali Pers.

Samsul, Mohamad, (2006), Pasar Modal dan Manajemen Portofolio, Jakarta :Erlangga

Sugiono, (2017), Metode Penelitian Kuantitatif, Kualitatif dan R\&D, Bandung: Alfabeta

www.bps.go.id, Publikasi Statistik Kelapa Sawit Indonesia

www.sahamok.com, Perusahaan Sub Sektor Perkebunan yang Listing

di Bursa Efek Indonesia, Tahun $2014-2018$.

www.idx.co.id, Laporan Keuangan Perusahaan Sub Sektor Perkebunan yang

Listing di Bursa Efek Indonesia, Tahun 2014-2018.

www.palmoilanalytics.com, Perkembangan Harga Crude Palm Oil (CPO) Dunia. 